\title{
Integrating Strategic and Tactical Rolling Stock Models with Cyclical Demand
}

\author{
Michael F. Gorman \\ Department of MIS, Operations and Decision Sciences, University of Dayton, \\ Dayton, USA \\ Email: Michael.gorman@udayton.edu
}

Received March 11, 2013; revised April 12, 2013; accepted April 20, 2013

Copyright (C) 2013 Michael F. Gorman. This is an open access article distributed under the Creative Commons Attribution License, which permits unrestricted use, distribution, and reproduction in any medium, provided the original work is properly cited.

\begin{abstract}
In the transportation industry, companies position rolling stock where it is likely to be needed in the face of a pronounced weekly cyclical demand pattern in orders. Strategic policies based on assumptions of repetition of cyclical weekly patterns set rolling stock targets; during tactical execution, a myriad dynamic influences cause deviations from strategically set targets. We find that optimal strategic plans do not agree with results of tactical modeling; strategic results are in fact suboptimal in many tactical situations. We discuss managerial implications of this finding and how the two modeling paradigms can be reconciled.
\end{abstract}

Keywords: Rolling Stock; Network Management; Strategic; Tactical

\section{Introduction}

Many freight transportation companies managing rolling stock fleets (e.g., containers, trailers, truck tractors, railcars, locomotives, etc.) face highly regular weekly cycles in supply of and demand patterns for these resources. For examples, supply and demand for rail locomotives may depend on the number of train terminations and originations, or in trucking, delivered loads contribute to container supply and historical order patterns indicate likely demand. These supply and demand vectors are heavily influenced by day of week (e.g., weekday versus weekend patterns). In this paper, we refer to "strategic" models as those based on this regular repeating patterns.

At a more tactical level, a transportation company must establish the best levels of rolling stock assets each day to support these highly cyclical and uncoordinated supply and demand patterns; transportation companies often keep non-zero levels of rolling stock capacity at locations in their network in anticipation of future demand because of the costs and time constraints of repositioning rolling stock. Because of the strong repeating weekly patterns of supply and demand, a company might develop target rolling stock levels based on strategic planning models that assume a regular weekly pattern to maximize the return on its rolling stock asset.

Although these regular patterns can be used for strategic planning, each week actual supply and demand levels vary around those patterns because of the stochastic nature of supply and demand, resulting in a deviation from the strategic plan. In this "tactical" setting, actual rolling stock inventory varies from the strategic targets; tactical models are deployed based on a starting condition forrolling stock levels.

In the tactical setting, in order to resolve such deviations and return to strategic target rolling stock levels, a company might make efforts to return to the optimal strategic inventory capacity levels such as increased or decreased allocation of the asset. However, the recovery or adjustment path often carries its own costs, so the company must assess if and when to adjust back to strategic targets.

Both strategic and tactical models have problems in implementation. The strategic model is difficult to manage in real time environment because of the assumption of cyclical repetition. The strategic model gives no indication how to react to deviations from the long run strategic optimum. On the other hand, strictly tactical modeling reflects current conditions in the network given prior events, and doesn't necessarily lead to any long run goals or targets. One intuitive solution in the tactical model paradigm is to start with current conditions, but at the end of the cycle, "recover", or return to the strategic target levels.

This research evaluates recovery strategies from a de- 
viation from the strategic target rolling stock levels and the appropriate integration of strategic and tactical models. We find that in some cases deviations from strategic optima are in fact advantageous; that is, the strategic optima may not be optimal in a tactical setting, calling into question the utility of strategic modeling of problems of this type. Managing to a target rolling stock level can be misdirected effort, creating additional costs with questionable incremental benefits. We evaluate conditions that give rise to this situation and make recommendations on how to reconcile the approaches. Based on these results, we make recommendations on the trade-offs between short run and long run rolling stock management.

\section{Literature}

The use of the words "strategic" and "tactical" require some definition. In some cases (e.g., [1]), strategic models focus on design of the transportation system, where tactical models focus on its operations. This is not the intended definition in this paper. Rather, we define a strategic model as one that assumes cyclicality, and a constraint on the ending time period ties it back to the starting time period. In this paper, "tactical" models start with a given starting condition, and may or may not have a constraint on the final period. In short, tactical models do not assume or require cyclicality. Because the two models have the same structure, and deal with the same issues in asset management, but differ only in assumption, their juxtaposition is warranted.

There are numerous examples of strategic and tactical models as defined above juxtaposed in the transportation literature. Similar to the strategic planning horizon described in [2] we define strategic patterns as those assuming a cyclic, repeating pattern that can be sustained in the long run, supporting a regular cycle and more strategic plan. An alternative to strategic planning is a more tactical orientation, which we call the tactical paradigm. Similar to the "daily" horizon of [2], we define tactical planning tools as have some initial (time $=t_{0}$ ) rolling stock inventory levels. In a similar way, [3], discusses various planning horizons in passenger rail: strategic, tactical, operational and short term. The tactical models of [3] are equivalent to the strategic models discussed here, and the operational models and short term planning models look at daily deviations as do these tactical models.

As depicted in Figure 1(a), strategic models include an arc from the end of the planning horizon, back to the start, imposing a repeatable cycle. In strategic models, ending period ending stock variables must equal the beginning period's starting value, thus creating continuity and consistency in the strategic model. Just as a circle must tie back to itself, so does a cyclic model's starting and ending inventory.
In the tactical model paradigm, the models are tied to a starting inventory condition (given all prior patterns of supply and demand and management allocation decisions, including unanticipated supply and demand shocks). Given a starting rolling stock inventory and anticipated cyclic supply and demand, what is the best course of action for managing these critical assets? Simply, tactical models react to current conditions which are a result of past known and exogenous events; Strategic models plan for them by viewing yesterday's events are next week's future events. Tactical models are necessary for dealing with a starting condition that are the result of prior events; strategic models are useful for establishing what the optimal conditions would be in the long run. The question addressed here is how to align these two modeling paradigms.

As depicted in Figure 1(b), tactical model might be specified with or without a constraint on the final state at the end of the horizon. On one hand, if there is a single deviation from the strategic target levels (say due to an unexpected supply or demand shock), the goal might be to manage from the current disequilibrium towards the "strategic target" level of inventory $\left(\mathrm{I}_{\mathrm{n}}=\mathrm{I}_{\mathrm{n}}^{\mathrm{s}}\right.$; dubbed "recovery" mode). Alternatively, the tactical setting might be more open-ended, with no constraint on ending inventory (dubbed tactical "reactionary" mode). The question remains, in the stochastic environment, how fast should adjustment take place if at all; how much weight should be given to strategic considerations? Secondarily, how close to strategic targets is "close enough", and what is the cost of deviating from this target? What is the cost of adjustment to the strategic optimum?

It is common in the literature to take either a strategic or tactical perspective on the problem without considering the alternative. For examples, [4-6] look at the challenge of managing railcars in the face of uncertain demand in the tactical setting, but do not consider a longerrun, strategic allocation of rolling stock or their optimal stocking levels. On the other hand, [7] considers fleet sizing under strategic assumptions, but do not discuss deviations from the strategic plans brought on by stochastic elements in a tactical, real time setting.

Similarly, [8] creates weekly repeating cycles of (strategic) locomotive to train assignments (and accompanying "ground arcs", or inventory decisions). On the other hand, [9] describes in-plant tactical locomotive manage-

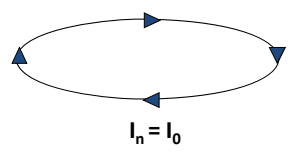

(a)

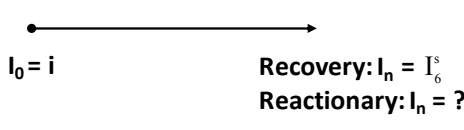

(b)
Figure 1. Conceptualization of strategic and tactical modeling paradigms. (a) Strategic modeling paradigm; (b) Tactical modeling paradigm. 
ment over a two-hour window with no consideration for longer-term considerations.

Similar to rolling stock, manpower capacity also must be managed by location. Reference [10] plans drayage operations and [11] plans drivers in a tactical setting, with a starting location for drivers and tractors specified, but because of short planning and order visibility horizons, make no consideration for repeatability or consistency of scheduling. Reference [12] discusses a tactical rail crew planning model given an initial starting location, and claims the model can be used for strategic manpower planning over a longer horizon, but does not show how to account for different starting conditions and stochastic train schedules.

A similar schism in focus can be found in the capacity pricing and yield management literature in freight transportation. For examples, [13] shows the importance of rolling stock balance in rail intermodal in a strategic setting, but does not talk about adjustment mechanisms in a tactical setting. Similarly, [14] builds a strategic logistics queueing network model that creates prices to place rolling stock capacity where and when it has the highest value. Reference [15], on the other hand, present a similar problem viewed from a tactical setting with a given starting inventory. There is no discussion in these articles on how to transition from a tactical yield management situation to the long run strategic pricing strategy, or how to translate strategic recommendations to a tactical implementation environment.

Reference [3] discusses decision horizon tradeoffs in passenger rail, which is considerably less volatile than freight rail systems, so short term planning models face similar constraints as air passenger service. However, [3] describes the different modeling paradigms, this research does not detail their differences nor try to reconcile them. It might be added that this research investigates the handoffs between the tactical and operational environments in the more uncertain freight transportation environment, where the differences of the two paradigms is more pronounced and the decisions on how to reconcile more difficult to address.

We should differentiate this research from the literature on "refleeting" or disruption management and recovery in the airline literature. This literature focuses on building robust cyclic schedules with respect to disruptions [16] and getting back on schedule in a least-cost way given a disruption [17]. In the airline case, there is a fixed schedule that must be followed per customer expectations and industry norms. This situation implies a required fleet capacity and mandatory and expedient recovery to the strategic condition; in the freight transporttation case, service provision depends on a stochastic order pattern without customer reservations with no obligation to follow specific schedule or level of capacity provision. The reason this distinction is important is that where airlines view the schedule as a constraint and recovery is mandatory, freight providers are not constrained to return to the strategic plan, but must choose both whether to recover, and how fast to return to the longer run strategic target levels.

In general, there is a dearth of literature which tries to bridge the gap in the planning process between strategic rolling stock planning and tacticalor real time execution in freight transportation. A notable counter example is an early attempt to meld tactical and strategic models in [18], which mitigates end effects in tactical vehicle allocation by proposing a "transient" (tactical) portion and a "stationary" (strategic) portion of the problem. With a discount factor, the repeating (looping) portion of the stationary portion represents the net present value of future flows. Reference [15] points out the managerial need for establishing target container inventory levels in intermodal, and identifies the problem surrounding the reconciliation of these two modeling paradigms, but does not resolve or make recommendations on how to resolve them. The contribution of this research is derived from its focus on the cost of deviation from the strategic plan, the cost of recovery, and derivation of appropriate recovery strategies. This research is the first to identify and quantify a contradiction between optimal strategies in strategic and tactical paradigms.

\section{Mathematical Modeling}

Below we provide a modeling construct which allows us to capture both strategic and tactical paradigms for comparative analysis. For simplicity of exposition and modeling, we will focus on the allocation of a single rolling stock inventory in a single location, but the results apply directly to the full multi commodity time space network. The model could be expanded to incorporate all locations in a transportation network, but for the purpose of this research, a single location model adequately demonstrates the point. Further, as noted in Gorman [15], the single node view matches the managerial focus of transportation companies managing tactical rolling stock inventories.

The decision variable, denoted $\mathrm{A}_{t}$, is the allocation of capacity of various types (tractors, drivers, locomotives, railcars, containers) to demands, $\mathrm{D}_{\mathrm{t}}$, of different types (trains, orders) in any period, t. The source of the allocated capacity in any period is based on the inventory of the resource carried from the previous period, $\mathrm{I}_{t}$, and that are made available from the supply process in that period, $\mathrm{S}_{\mathrm{t}}$. In the tactical setting, supply and demand are considered exogenous. Demand is exogenous based on customer order patterns. Supply is exogenous because it is the result of terminated usage from past allocation decisions in the tactical setting and because it is the result of 
allocation decisions made in other geographic locations in both the tactical and strategic modeling paradigms.

The allocation of each asset depends on its cost and revenue profiles in various uses. The explicit cost of excess inventory is higher inventory carrying costs. The opportunity cost of excess rolling stock is the acceptance of lower profitability business (higher cost or lower revenue) in order to utilize the asset.

The cost of high inventory must be balanced against the opportunity cost of low inventory levels. In the single asset case, the primary opportunity cost of a rolling stock inventory shortage is lost revenue. In the multiple asset case, a shortage of a preferred asset requires the use of a less preferred alternative-either a lower revenue or higher cost asset. Different asset classes which are imperfect substitutes, with "preferred" and "less preferred" assignment which constitute varying cost profiles and capabilities which govern the feasibility of their assignment. For examples, locomotives of 4 and 6 axles have different fuel efficiencies, tractive effort potential, or consist interoperability, making them have different efficiency levels for different train types [8]. Different railcars might have different equipment rent if they are foreign owned or not [15]. Drivers may be different distances from an order, requiring varying dray costs. Containers of different ownership have different cost structures and rail routing options [15]. In each case, there is some incremental cost of using the imperfect substitute for a given demand. From a revenue perspective, different sizes of containers or equipment on railcars might have diminishing values to customers, or from a safety stock perspective, additional inventory of any kind has a diminishing but positive probability of use, so incremental units of capacity have diminishing expected revenue [15].

As discussed in [15] such opportunity costs are likely to be increasing as the level of surplus or shortage grows. Both the incremental costs and diminishing revenues contribute to a decreasing valuation of any asset at a location as the quantity of that asset grows. We define the "profit advantage function", asy * $\left(\mathrm{x}-\mathrm{A}_{\mathrm{t}} / \mathrm{D}_{\mathrm{t}}\right)^{\mathrm{z}}$, of one class of rolling stock inventory over another to capture this diminishing profitability relationship. (This is simply the profit function in the case with only one asset class.) $A_{t} / D_{t}$ is the percentage of total demand on day $t$ allocated to asset $\mathrm{A}$ on day $\mathrm{t}\left(0 \leq \mathrm{A}_{\mathrm{t}} \leq \mathrm{D}_{\mathrm{t}}\right)$. A generalized diminishing profitability function with parameters $\mathrm{x}, \mathrm{y}$, and $\mathrm{z}$ is specified in order to test for sensitivity of our results for different functional forms of the profit equation: y specifies the highest valuation of the most appropriate allocation, $x$ determines the percentage of total allocation at which the expected contribution becomes negative, and $z$ indicates the concavity $(z>1)$, convexity $(z<1)$ or linearity $(z=1)$ of the functional form. Such a relationship is easily estimated from historical data on assignments and profitability. Examples of each functional form are demonstrated in Figure 2.

\section{Optimization Model}

The optimization model that serves as the basis of our study is described in Equations (1)-(4). The profit advan-

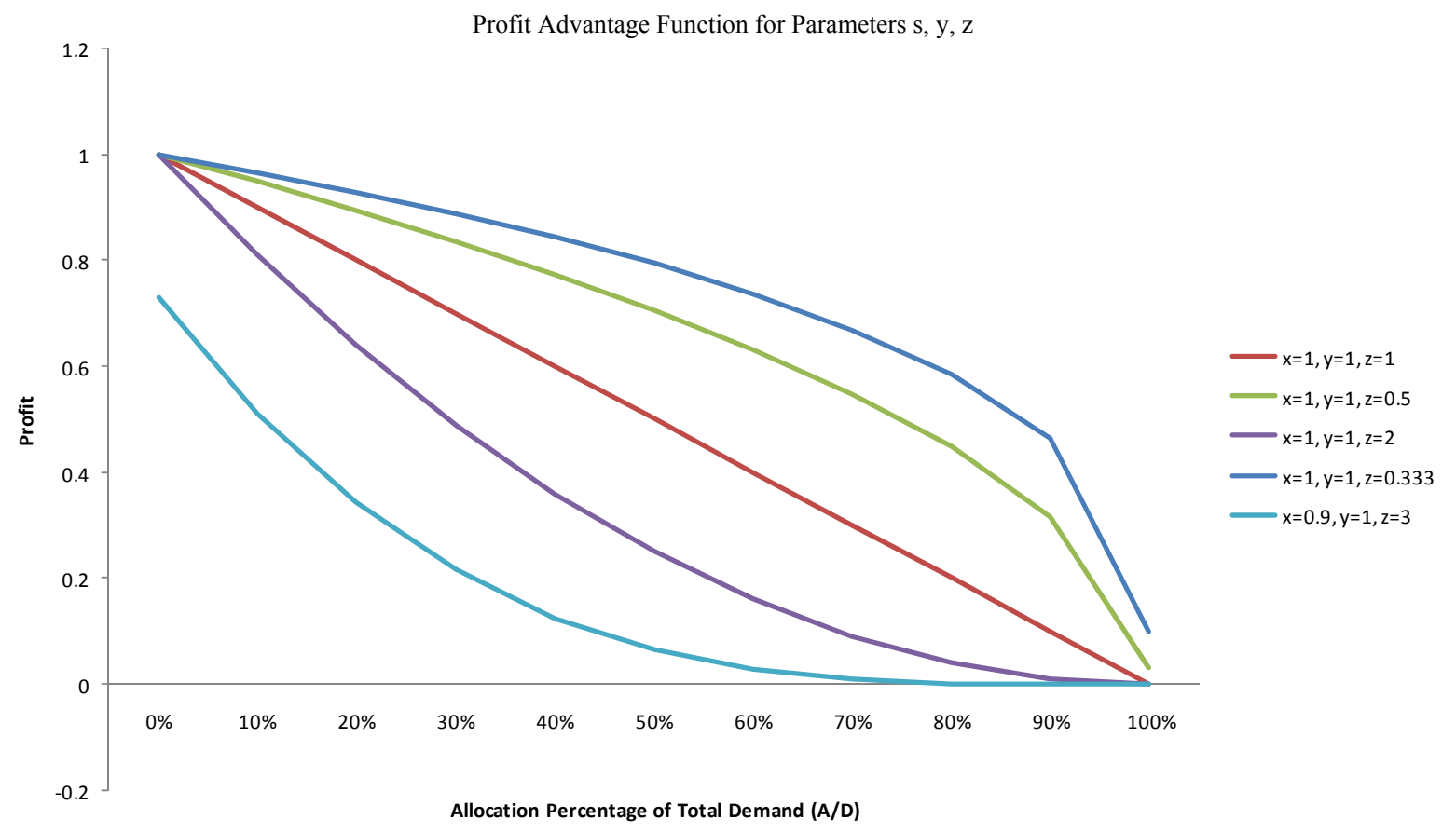

Figure 2. Profit advantage function for parameters s, y, z. 
tage function is the first component of the objective function (Equation (1)). A secondary disadvantage of carrying inventory, $\mathrm{I}_{\mathrm{t}}$, of some asset class is its holding cost, $\mathrm{HC}$, which is subtracted from the expected profitability of each assignment level, the second component of Equation (1).

The constraints governing assignments are given in Equations (2)-(4). Allocations of an asset must be less than demand (2), inventory in any period equals the inventory of the prior period, plus new supply, less allocation in this period (3), and inventory must be non-negative.

Maximize

$$
p^{*}=y\left(x-D_{t} / A_{t}\right)^{z}-\sum_{t=1}^{7} H C I_{t}
$$

Subject to:

$$
\begin{aligned}
& 0 \leq \mathrm{A}_{\mathrm{t}} \leq \mathrm{D}_{\mathrm{t}} \mathrm{t} \forall \mathrm{t} \\
& I_{\mathrm{t}+1}=S_{\mathrm{t}+1}-A_{\mathrm{t}+1}+\mathrm{I}_{\mathrm{t}} \forall \mathrm{t} \\
& I_{\mathrm{t}} \geq 0 \forall \mathrm{t}
\end{aligned}
$$

Without loss of generality, we focus on the weekly supply and demand paper with seven daily time periods, t. Constraints $5 \mathrm{~s}$ and $5 \mathrm{~d}$ differentiate the strategic and tactical modeling paradigms. In the strategic paradigm, starting inventory is related ending inventory to assure cyclic repeatability:

$$
\mathrm{I}_{1}=\mathrm{I}_{7}+\mathrm{S}_{1}-\mathrm{A}_{1}
$$

We define the resulting profit to the strategic problem given in Equations (1)-(5s) as $\mathrm{p}^{\mathrm{s}}$, optimal allocations vector as $A_{t}^{s}$, and resulting inventory vector as $I_{t}^{s}$.

In the tactical model, constraint $5 \mathrm{~s}$ is replaced with $5 \mathrm{~d}$ :

$$
\mathrm{I}_{1}^{d}=\mathrm{I}_{0} 0+\mathrm{S}_{1}-\mathrm{A}_{1}
$$

where $\mathrm{I}_{0}$ is some initial, exogenous inventory value in the tactical setting given past supply and demand shocks.

Within the tactical paradigm, management might not constrain ending inventory, $\mathrm{I}_{7}$, following a reactionary, short term tactical (t) strategy, choosing to react to supply and demand perturbations with a short term focus, disregarding the strategic optimum. We denote objective function values, inventory levels and allocations as $\mathrm{p}^{\mathrm{t}}, \mathrm{I}^{\mathrm{t}}$ and $\mathrm{A}^{\mathrm{t}}$. Alternatively, management might pursue what we will call a "recovery" (r) strategy that allows them to regain strategic inventory levels by constraining ending inventory to equal that of the strategic model as in Equation (6).

$$
\mathrm{I}_{7}^{r}=\mathrm{I}_{7}^{\mathrm{s}} .
$$

We denote objective function values, inventory levels and allocations as $\mathrm{p}^{\mathrm{r}}, \mathrm{I}^{\mathrm{r}}$ and $\mathrm{A}^{\mathrm{r}}$. For any given $\mathrm{D}$ and $\mathrm{S}$ arrays, each of these three models lead to different values of the decision variables, inventory and total objective function values.

\section{Numerical Example}

We illustrate the optimization models with a numerical example. In this illustration, key input parameters are: $\mathrm{HC}=0.1, \mathrm{x}=\mathrm{y}=\mathrm{z}=1$. We select a random demand (D) and supply (S) arrays as depicted in Table 1, and solving the strategic, reactionary and tactical optimization models given in Equations (1)-(6), results in optimal profitability (p), fleet allocations (A) and inventory levels (I) under each paradigm displayed in the columns as labeled. In the strategic model, $\mathrm{I}_{0}$ is based on $\mathrm{I}_{7}$ at the end of the week, thus is endogenously determined. In this example, the optimal end-of-week inventory is 7 units to achieve a strategic sustainable profit of $\$ 72.74$ per week. If a manager was myopic or did not put emphasis on the somewhat uncertain future supply and demand patters, he would ignore end-of-week inventory on the subsequent week's profits (the constraint that $\mathrm{I}_{0}=\mathrm{I}_{7}$ is removed). As a result, $\$ 74.67$ could be earned in a week. However, the ending inventory of 0 units $\left(I_{7}=I_{0}=0\right)$ in reactionary mode drives the subsequent week's profit down to $\$ 69.29$ if the goal is to "recover"-return to the strategic optimum inventory $\left(\mathrm{I}_{7}=7\right)$ - by the end of the second week (as shown in the last column of Table 1, in which starting inventory is zero and ending inventory is seven). Average profits fall from a strategic expectation of $\$ 72.74$, to an average of $\$ 71.98=(\$ 74.67+69.29) / 2$. Thus, unsustainable short-term profit is gained at the expense of future recovery costs. It seems reasonable, then, to strive to achieve strategic target inventories.

However, deviations from the strategic optimum could be for exogenous reasons no fault of the manager, such as an unanticipated supply or demand shock. Let us assume a single a priori exogenous supply shock leads to some deviation from strategic optimum inventory at the end of Day 0. In this case, the manager optimizes given some starting inventory level. The manager has a choice to try to recover to the strategic target inventory or not.

We solved the tactical model to optimality for reasonable levels of starting inventory ranging from zero to 59 results in a quadratic shaped profit curve as indicated in Figure 3. While the strategic optimum (and the recovery tactical target ending inventory) starting inventory is seven, profitability in a given week is maximized at a starting inventory of 25 units, resulting in $\$ 4.82(6.6 \%)$ in higher profits from the presence of that inventory with no recovery to strategic targets, and $\$ 2.89(2.6 \%)$ increased profits if recovery to strategic is completed by day seven. Although such profit is not sustainable in the long run if the strategic supply and demand processes are representative of normal patterns, these results call into 
Table 1. Number example of state optimal inventory, and dynamic reactionary and dynamic recovery profit levels.

\begin{tabular}{|c|c|c|c|c|c|c|c|c|c|c|c|c|c|c|c|c|c|c|}
\hline & & & \multicolumn{4}{|c|}{$\begin{array}{c}\text { Steady State Model } \\
\left(\mathbf{I}_{0}=\mathbf{I}_{7} ; \mathbf{I}_{7} \text { is a decision }\right. \\
\text { variable })\end{array}$} & \multicolumn{4}{|c|}{$\begin{array}{c}\text { Dynamic Reactionary Model } \\
\left(\mathbf{I}_{0}=\mathbf{I}_{7}^{\mathrm{s}}=7 ; \mathbf{I}_{7} \text { unconstrained }\right)\end{array}$} & \multicolumn{4}{|c|}{$\begin{array}{l}\text { Dynamic Recovery Model } \\
\left(\mathbf{I}_{0}=\mathbf{I}_{7}^{\mathrm{r}}=\mathbf{0} ; \mathbf{I}_{7}^{\mathrm{s}}=\mathbf{7}\right)\end{array}$} & \multicolumn{4}{|c|}{$\begin{array}{c}\text { Optimal Starting } \\
\text { Tactical Inventory } \\
\text { (I } \mathbf{I}_{0}=0 ; \mathbf{I}_{7}^{\mathbf{s}}= \\
\text { Unconstrained) }\end{array}$} \\
\hline & \multicolumn{2}{|c|}{ 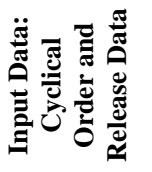 } & 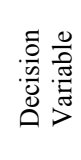 & & 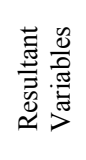 & & 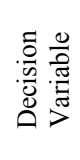 & & 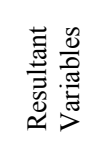 & & 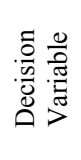 & & 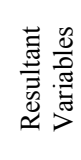 & & \multicolumn{2}{|l|}{ 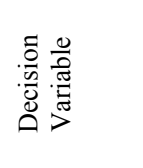 } & \multicolumn{2}{|l|}{ 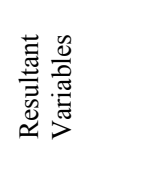 } \\
\hline ఫ્ञ & 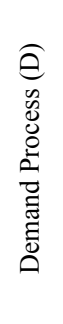 & 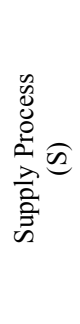 & 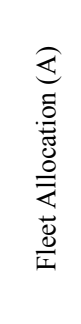 & 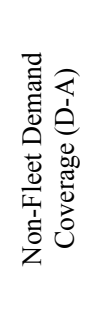 & 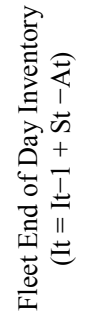 & 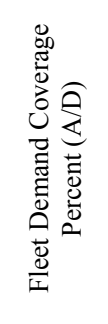 & 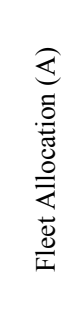 & 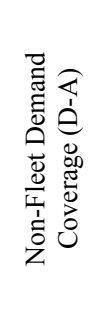 & 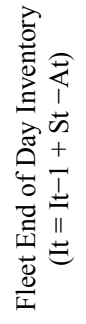 & 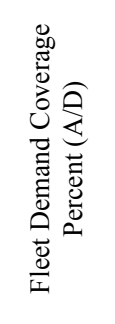 & 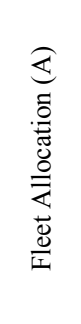 & 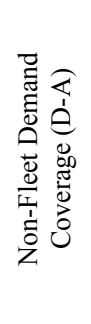 & 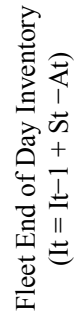 & 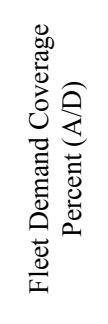 & 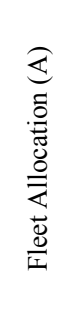 & 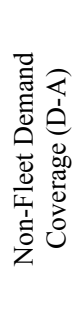 & 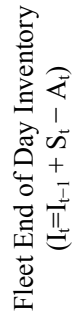 & 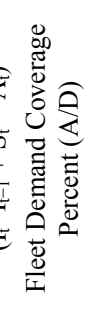 \\
\hline \multicolumn{3}{|c|}{$\begin{array}{l}\text { End } \\
\text { Day } 0\end{array}$} & \multicolumn{4}{|c|}{7} & \multicolumn{4}{|c|}{7} & \multicolumn{4}{|c|}{ - } & \multicolumn{4}{|c|}{25} \\
\hline 1 & 54 & 10 & 17 & 37 & - & $31 \%$ & 17 & 37 & - & $31 \%$ & 10 & 44 & - & $19 \%$ & 27 & 27 & 8 & $50 \%$ \\
\hline 2 & 51 & 16 & 16 & 35 & - & $31 \%$ & 16 & 35 & - & $31 \%$ & 16 & 35 & - & $31 \%$ & 23 & 28 & 1 & $45 \%$ \\
\hline 3 & 20 & 18 & 8 & 12 & 10 & $40 \%$ & 8 & 12 & 10 & $40 \%$ & 8 & 12 & 10 & $40 \%$ & 8 & 12 & 11 & $40 \%$ \\
\hline 4 & 87 & 20 & 30 & 57 & - & $35 \%$ & 30 & 57 & - & $35 \%$ & 30 & 57 & - & $35 \%$ & 31 & 56 & - & $35 \%$ \\
\hline 5 & 15 & 21 & 7 & 8 & 14 & $46 \%$ & 7 & 8 & 14 & $49 \%$ & 7 & 8 & 14 & $46 \%$ & 7 & 8 & 14 & $49 \%$ \\
\hline 6 & 76 & 20 & 31 & 45 & 3 & $41 \%$ & 34 & 42 & - & $44 \%$ & 31 & 45 & 3 & $41 \%$ & 34 & 42 & - & $44 \%$ \\
\hline 7 & 30 & 15 & 11 & 19 & 7 & $36 \%$ & 15 & 15 & - & $50 \%$ & 11 & 19 & 7 & $36 \%$ & 15 & 15 & - & $50 \%$ \\
\hline \multicolumn{3}{|c|}{$\begin{array}{l}\text { Input Fleet Profit } \\
\text { Parameters }\end{array}$} & \multicolumn{4}{|c|}{$\begin{array}{c}\text { Fleet } \\
\text { Proftibality (p) }\end{array} 76.12$} & \multicolumn{4}{|c|}{$\begin{array}{c}\text { Fleet } \\
\text { Proftibality (p) }\end{array}$} & \multicolumn{4}{|c|}{$\begin{array}{c}\text { Fleet } \\
\text { Proftibality (p) }\end{array} 72.67$} & \multirow{2}{*}{\multicolumn{3}{|c|}{$\begin{array}{c}\text { Fleet } \\
\text { Proftibality } \\
(p) \\
\text { Less Fleet } \\
\text { inventory } \\
\text { cost }\end{array}$}} & $\begin{array}{c}\$ 80.8 \\
1\end{array}$ \\
\hline $\mathrm{x}$ & 1 & & \multicolumn{3}{|c|}{$\begin{array}{c}\text { Less Fleet } \\
\text { inventory cost }\end{array}$} & $\$ 3.37$ & \multicolumn{3}{|c|}{$\begin{array}{c}\text { Less Fleet } \\
\text { inventory cost }\end{array}$} & $\$ 2.37$ & \multicolumn{3}{|c|}{$\begin{array}{c}\text { Less Fleet } \\
\text { inventory cost }\end{array}$} & $\$ 3.37$ & & & & $\$ 3.29$ \\
\hline $\mathrm{x}$ & \multicolumn{2}{|l|}{1} & \multirow{2}{*}{\multicolumn{3}{|c|}{$\begin{array}{l}\text { Net Fleet } \\
\text { Benefit }\end{array}$}} & $\$ 72.74$ & \multirow{2}{*}{\multicolumn{3}{|c|}{$\begin{array}{l}\text { Net Fleet } \\
\text { Benefit }\end{array}$}} & $\$ 74.67$ & \multirow{2}{*}{\multicolumn{3}{|c|}{$\begin{array}{l}\text { Net Fleet } \\
\text { Benefit }\end{array}$}} & $\$ 69.29$ & \multirow{2}{*}{\multicolumn{3}{|c|}{$\begin{array}{l}\text { Net Fleet } \\
\text { Benefit }\end{array}$}} & $\begin{array}{c}\$ 77.5 \\
2\end{array}$ \\
\hline $\mathrm{z}$ & 1 & & & & & & & & & & & & & & & & & \\
\hline $\mathrm{HC}$ & 0.1 & & & & & & & & & & & & & & & & & \\
\hline
\end{tabular}

question whether deviations from strategic targets are in fact bad, and if strenuous efforts should be made to return to strategic targets. Similar unanticipated supply and demand shocks over the planning horizon could render striving for strategic targets both infeasible and unprofitable. In a highly stochastic environment, the shortsighted manager who discounts the future states might actually achieve superior results.

\section{Monte Carlo Simulation}

Of course, this example could be a special case. To investigate how wide spread this result is, we conducted numerical experiments based on a Monte Carlo simulation. The algorithm below compares the strategic opti- mum profit with the profit level consistent with the optimal tactical inventory level. To compare the cost of deviation from target levels, we solve both models repeatedly in $n$ Monte Carlo replications of weekly $\mathrm{S}$ and $\mathrm{D}$ patterns. We then constrain $\mathrm{I}_{0}$ for all reasonable levels of inventory $\left(\mathrm{I}_{\max }\right)$ to evaluate the change in cost from the optimal strategic level $\mathrm{p}^{\mathrm{s}}$ from dynamic model objective function values $p^{r}$ and $p^{t}$. Importantly, we compare the deviation from optimal $p_{s}$ profit levels for each starting level of I.

The approach takes three general steps:

1) For any random generation of $S$ and $D$ arrays, solve the strategic model to establish long run targets (s);

2) Perform sensitivity analysis with respect to devia- 


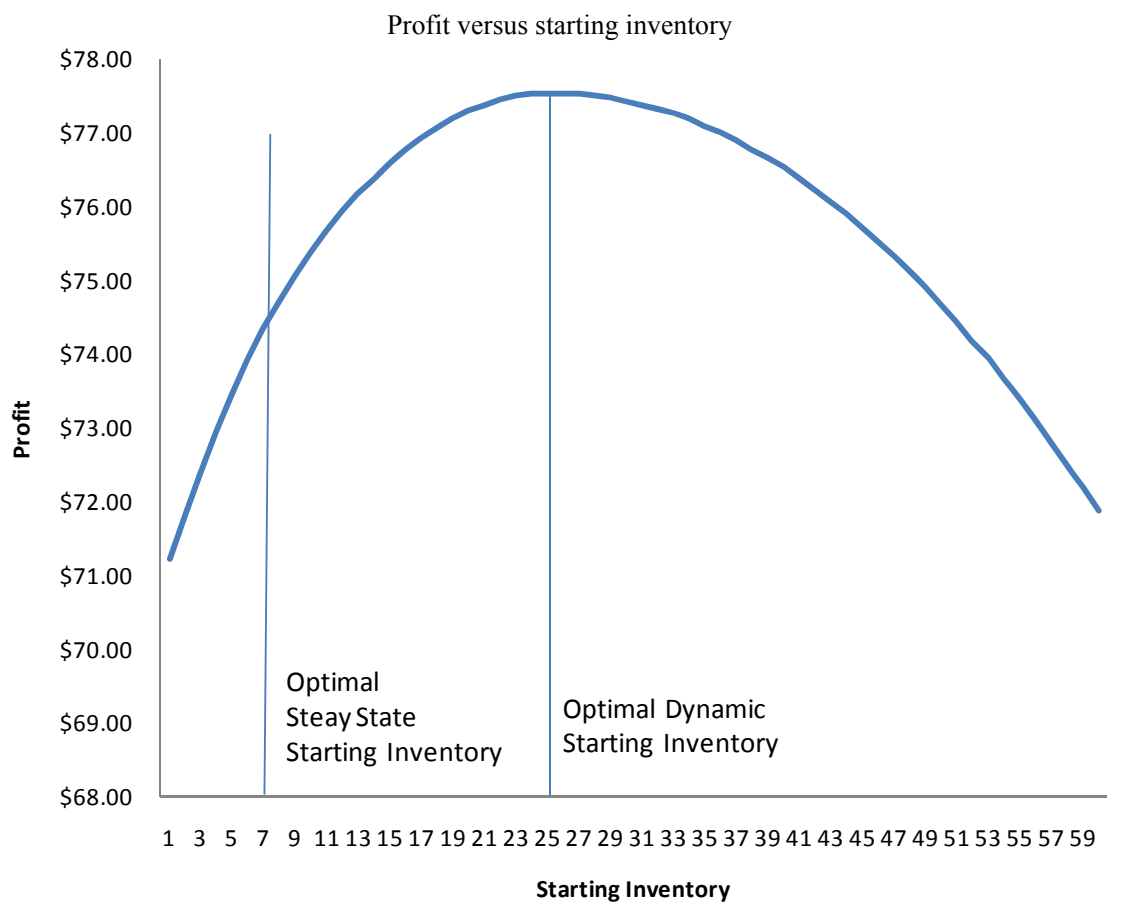

Figure 3. Profit versus starting inventory.

tions from optimum starting inventory. For all reasonable starting inventory levels, $\mathrm{I}_{0}$, solve tactical model twice, once in recovery mode (r), once in tactical reactive mode (t), comparing tactical model objective function values with that of strategic model.

3) The difference between the optimal based on strategic target and optimal based on current tactical inventory is the objective function loss or gain from deviating from the strategic optimum.

The algorithm below describes the steps in more detail.

For $\mathrm{j}=1$ to $\mathrm{n}$

Generate random replications for $\mathrm{S}$ and $\mathrm{D}$ processes

Solve strategic model for $\mathrm{p}^{\mathrm{s}}, \mathrm{I}^{\mathrm{s}}, \mathrm{A}^{\mathrm{s}}$

For $\mathrm{i}=0$ to $\mathrm{I}_{\max }$

$\mathrm{I}_{0}=\mathrm{i}$

Solve the tactical model for $\mathrm{p}^{\mathrm{r}}, \mathrm{I}^{\mathrm{r}}, \mathrm{A}^{\mathrm{r}}$

DeviationCost ${ }^{\mathrm{r}}=\mathrm{p}^{\mathrm{s}}-\mathrm{p}^{\mathrm{r}}$

Solve the tactical model for $\mathrm{p}^{\mathrm{t}}, \mathrm{I}^{\mathrm{t}}, \mathrm{A}^{\mathrm{t}}$

DeviationCost ${ }^{t}=p^{s}-p^{t}$

Next i

Next j

\subsection{Experimental Design}

We set up a balanced experiment with 12 scenarios: 2 variance levels (High, Low), 3 supply/demand ratio levels (High, Medium and Low supply), and 3 functional forms (linear, concave and convex). In the structured experiments, we held three parameters constant: $\mathrm{x}=1, \mathrm{y}$ $=33 *$ HC. For the high supply and demand variance, a uniform distribution was used to generate supply and demand vectors, $\mathrm{D} \sim \mathrm{U}(1,100)$; for the low supply and demand variance, a Poisson distribution was used, D Poisson(50). For high supply, the expected value of $\mathrm{S}=$ $0.5 \mathrm{D}$, for medium supply, $\mathrm{S}=0.4 \mathrm{D}$, and for low supply, $\mathrm{S}=0.33 \mathrm{D}$. Strategic model results reflect a single model run; tactical model results reflect the tactical model run with the best starting inventory (minimum deviation cost from strategic optimum). We conducted 40 randomly generated replications for each scenario. For the functional form of the profit advantage function, we set $\mathrm{z}=2$ (convex), 1 (linear) and 0.5 (concave). Descriptive statistics are presented in Table 2.

We can see from Table 2, no matter what the functional form, variance or supply demand ratio, the average objective based on ideal tactical starting inventory is always higher than the strategic optimum. That is, there is some level of inventory other than the strategic optimum which makes the objective increase, over the decision horizon. This effect is generally more pronounced in the high variance scenarios. Simply, the strategic, strategic optimum is not optimal in a tactical setting.

Further, by comparing the tactical-reactionary objective function value to the tactical-recovery objective, we see that recovery has a cost; by working to return to some steady-state ideal inventory by the end of the week, profit potential is lost. It is worth mentioning that the profit performance of the reactionary modeling paradigm may not be sustainable because the end of the week inventory levels may not support future business patterns well, but 
Table 2. Comparison of objective function values in stratified random experiments.

\begin{tabular}{|c|c|c|c|c|c|c|c|}
\hline \multirow[t]{3}{*}{ Linear } & & \multicolumn{6}{|c|}{ Objective Function Values } \\
\hline & & \multicolumn{2}{|c|}{ Strategic } & \multicolumn{2}{|c|}{ Tactical-Reactionary } & \multicolumn{2}{|c|}{ Tactical-Recovery } \\
\hline & & Mean & Std Dev & Mean & Std Dev & Mean & Std Dev \\
\hline HSHVLIN & High Supply, High Var, Linear & $\$ 29,701$ & $\$ 11,478$ & $\$ 32,591$ & $\$ 8282$ & $\$ 31,359$ & $\$ 10,188$ \\
\hline MSHVLIN & Medium Supply, High Var, Linear & $\$ 37,084$ & $\$ 7555$ & $\$ 39,131$ & $\$ 7557$ & $\$ 38,200$ & $\$ 7770$ \\
\hline LSHVLIN & Low Supply, High Var, Linear & $\$ 42,821$ & $\$ 8560$ & $\$ 46,200$ & $\$ 8211$ & $\$ 44,792$ & $\$ 8536$ \\
\hline HSLVLIN & High Supply, Low Var, Linear & $\$ 38,449$ & $\$ 3125$ & $\$ 38,721$ & $\$ 3194$ & $\$ 38,328$ & $\$ 3338$ \\
\hline MSLVLIN & Medium Supply, Low Var, Linear & $\$ 47,333$ & $\$ 2311$ & $\$ 48,595$ & $\$ 2582$ & $\$ 48,365$ & $\$ 2533$ \\
\hline LSLVLIN & Low Supply, Low Var, Linear & $\$ 52,093$ & $\$ 2688$ & $\$ 55,429$ & $\$ 3012$ & $\$ 55,007$ & $\$ 3037$ \\
\hline Average & Average & $\$ 41,247$ & $\$ 9962$ & $\$ 43,445$ & $\$ 9595$ & $\$ 42,675$ & $\$ 10,130$ \\
\hline \multirow[t]{3}{*}{ Concave } & & \multicolumn{6}{|c|}{ Objective Function Values } \\
\hline & & \multicolumn{2}{|c|}{ Strategic } & \multicolumn{2}{|c|}{ Tactical-Reactionary } & \multicolumn{2}{|c|}{ Tactical-Recovery } \\
\hline & & Mean & Std Dev & Mean & Std Dev & Mean & Std Dev \\
\hline HSHVCAVE & High Supply, High Var, Concave & $\$ 44,996$ & $\$ 16,983$ & $\$ 49,366$ & $\$ 14,501$ & $\$ 47,814$ & $\$ 15,396$ \\
\hline MSHVCAVE & Medium Supply, High Var, Concave & $\$ 53,432$ & $\$ 10,964$ & $\$ 59,134$ & $\$ 10,648$ & $\$ 56,805$ & $\$ 10,903$ \\
\hline LSHVCAVE & Low Supply, High Var, Concave & $\$ 58,131$ & $\$ 14,223$ & $\$ 64,313$ & $\$ 12,215$ & $\$ 62,401$ & $\$ 13,334$ \\
\hline HSLVCAVE & High Supply, Low Var, Concave & $\$ 66,596$ & $\$ 3,451$ & $\$ 71,860$ & $\$ 4,156$ & $\$ 71,202$ & $\$ 4,039$ \\
\hline MSLVCAVE & Medium Supply, Low Var, Concave & $\$ 62,281$ & $\$ 3,048$ & $\$ 65,348$ & $\$ 3,383$ & $\$ 64,780$ & $\$ 3,286$ \\
\hline LSLVCAVE & Low Supply, Low Var, Concave & $\$ 58,267$ & $\$ 4,223$ & $\$ 59,898$ & $\$ 4,418$ & $\$ 59,542$ & $\$ 4,477$ \\
\hline Grand Total & Average & $\$ 57,284$ & $\$ 12,361$ & $\$ 61,653$ & $\$ 11,523$ & $\$ 60,424$ & $\$ 12,124$ \\
\hline & & & & Objective Fun & in Values & & \\
\hline \multirow[t]{2}{*}{ Convex } & & \multicolumn{2}{|c|}{ Strategic } & \multicolumn{2}{|c|}{ Tactical-Reactionary } & \multicolumn{2}{|c|}{ Tactical-Recovery } \\
\hline & & Mean & Std Dev & Mean & Std Dev & Mean & Std Dev \\
\hline HSHVCAVE & High Supply, High Var, Convex & $\$ 14,510.63$ & $\$ 10,279.24$ & $\$ 16,246.75$ & $\$ 7789.73$ & $\$ 15,464.76$ & $\$ 8856.95$ \\
\hline MSHVCAVE & Medium Supply, High Var, Convex & $\$ 25,155.82$ & $\$ 5988.79$ & $\$ 26,010.81$ & $\$ 6377.54$ & $\$ 25,848.05$ & $\$ 6309.42$ \\
\hline LSHVCAVE & Low Supply, High Var, Convex & $\$ 26,019.86$ & $\$ 7343.24$ & $\$ 26,973.27$ & $\$ 7779.07$ & $\$ 26,594.44$ & $\$ 7634.80$ \\
\hline HSLVCAVE & High Supply, Low Var, Convex & $\$ 22,919.27$ & $\$ 1593.77$ & $\$ 22,983.77$ & $\$ 1573.92$ & $\$ 22,940.86$ & $\$ 1594.72$ \\
\hline MSLVCAVE & Medium Supply, Low Var, Convex & $\$ 25,722.16$ & $\$ 1766.67$ & $\$ 25,763.86$ & $\$ 1777.73$ & $\$ 25,750.90$ & $\$ 1776.19$ \\
\hline LSLVCAVE & Low Supply, Low Var, Convex & $\$ 28,307.34$ & $\$ 1698.89$ & $\$ 28,434.25$ & $\$ 1736.51$ & $\$ 28,395.70$ & $\$ 1729.95$ \\
\hline Grand Total & Average & $\$ 23,958.98$ & $\$ 6682.89$ & $\$ 24,436.24$ & $\$ 5915.60$ & $\$ 24,246.38$ & $\$ 6259.72$ \\
\hline
\end{tabular}

this risk must be balanced with the reward of enhanced high probability short term profit.

A more direct comparison of the three models can be conducted by looking at the foregone profit under each random demand generation. In each replication, we calculated the absolute increase in profits between the modeling paradigms objective function values. Over $90 \%$ of the time, the differences were non-zero, demonstrating the modeling paradigm and resulting operating policy does make a difference to profits under most supply and demand conditions. Table 3 presents the differences in mean profit levels under each modeling paradigm and scenario. We see that there is a starting inventory level in the tactical setting that leads to an average of $5.5 \%$ higher profits that the inventory suggested by the strategic model. Further, an average of $1.8 \%$ profits are fore- gone when striving to recover to the strategically identified optimum rather than simply focusing on the near term. In total, $7.3 \%$ profits are lost by focusing on a strategically identified optimum that is inappropriate in a tactical, execution setting.

\subsection{Completely Randomized Experimental Design}

To ensure robustness, we also ran a completely randomized design experiment that varied $\mathrm{x}, \mathrm{y}, \mathrm{z}$ and $\mathrm{HC}: \mathrm{x}$ $\mathrm{U}(0.5,1), \mathrm{y} \sim \mathrm{U}(100,500), \mathrm{z}=\mathrm{U}(1,3), \mathrm{HC} \sim \mathrm{U}(5,30)$ with both the medium and high supply, and with low and high variance. We generated 250 replications in this completely randomized design. Although the standard deviation of the profit differentials was higher due to the more varied input data, the results from the stratified 
Table 3. Difference in mean profit levels between modeling paradigms.

\begin{tabular}{|c|c|c|c|c|c|}
\hline \multicolumn{6}{|l|}{ Linear } \\
\hline & & \multicolumn{2}{|c|}{ Tactical Recov-Strategic } & \multicolumn{2}{|c|}{ Tactical React-Recovery } \\
\hline & & Abs. Diff & Pct. Diff & Abs. Diff & Pct. Diff \\
\hline HSHVLIN & High Supply, High Var, Linear & $\$ 2891$ & $8.9 \%$ & $\$ 1232$ & $3.9 \%$ \\
\hline MSHVLIN & Medium Supply, High Var, Linear & $\$ 2048$ & $5.2 \%$ & $\$ 931$ & $2.4 \%$ \\
\hline LSHVLIN & Low Supply, High Var, Linear & $\$ 3379$ & $7.3 \%$ & $\$ 1408$ & $3.1 \%$ \\
\hline HSLVLIN & High Supply, Low Var, Linear & $\$ 272$ & $0.7 \%$ & $\$ 393$ & $1.0 \%$ \\
\hline MSLVLIN & Medium Supply, Low Var, Linear & $\$ 1262$ & $2.6 \%$ & $\$ 230$ & $0.5 \%$ \\
\hline LSLVLIN & Low Supply, Low Var, Linear & $\$ 3336$ & $6.0 \%$ & $\$ 423$ & $0.8 \%$ \\
\hline Average & Average & $\$ 2198$ & $5.1 \%$ & $\$ 769$ & $2.0 \%$ \\
\hline \multicolumn{6}{|l|}{ Concave } \\
\hline & & \multicolumn{2}{|c|}{ Tactical Recov-Strategic } & \multicolumn{2}{|c|}{ Tactical React-Recovery } \\
\hline & & Abs. Diff & Pct. Diff & Abs. Diff & Pct. Diff \\
\hline HSHVCAVE & High Supply, High Var, Concave & $\$ 4370$ & $8.9 \%$ & $\$ 1553$ & $3.2 \%$ \\
\hline MSHVCAVE & Medium Supply, High Var, Concave & $\$ 5702$ & $9.6 \%$ & $\$ 2329$ & $4.1 \%$ \\
\hline LSHVCAVE & Low Supply, High Var, Concave & $\$ 6181$ & $9.6 \%$ & $\$ 1912$ & $3.1 \%$ \\
\hline HSLVCAVE & High Supply, Low Var, Concave & $\$ 5264$ & $7.3 \%$ & $\$ 657$ & $0.9 \%$ \\
\hline MSLVCAVE & Medium Supply, Low Var, Concave & $\$ 3068$ & $4.7 \%$ & $\$ 569$ & $0.9 \%$ \\
\hline LSLVCAVE & Low Supply, Low Var, Concave & $\$ 1631$ & $2.7 \%$ & $\$ 357$ & $0.6 \%$ \\
\hline Grand Total & Average & $\$ 4369$ & $7.1 \%$ & $\$ 1229$ & $2.1 \%$ \\
\hline \multirow[t]{2}{*}{ Convex } & & \multicolumn{2}{|c|}{ Tactical Recov-Strategic } & \multicolumn{2}{|c|}{ Tactical React-Recovery } \\
\hline & & Abs. Diff & Pct. Diff & Abs. Diff & Pct. Diff \\
\hline HSHVCAVE & High Supply, High Var, Convex & $\$ 1736$ & $10.7 \%$ & $\$ 782$ & $5.1 \%$ \\
\hline MSHVCAVE & Medium Supply, High Var, Convex & $\$ 855$ & $3.3 \%$ & $\$ 163$ & $0.6 \%$ \\
\hline LSHVCAVE & Low Supply, High Var, Convex & $\$ 953$ & $3.5 \%$ & $\$ 379$ & $1.4 \%$ \\
\hline HSLVCAVE & High Supply, Low Var, Convex & $\$ 64$ & $0.3 \%$ & $\$ 43$ & $0.2 \%$ \\
\hline MSLVCAVE & Medium Supply, Low Var, Convex & $\$ 42$ & $0.2 \%$ & $\$ 13$ & $0.1 \%$ \\
\hline LSLVCAVE & Low Supply, Low Var, Convex & $\$ 127$ & $0.4 \%$ & $\$ 39$ & $0.1 \%$ \\
\hline Grand Total & Average & $\$ 630$ & $3.1 \%$ & $\$ 236$ & $1.2 \%$ \\
\hline
\end{tabular}

experiment were supported; in every case there was an inventory level in a tactical setting that was preferred to the strategic, stead state optimum, and any effort of inventory recovery to the strategic levels incurred a cost to the objective function. The mean profit improvement of fortuitous tactical inventory deviations from strategic levels is $\$ 491.67(2.0 \%)$ with a standard deviation of 1239.3. Inventory level recovery costs on average $\$ 544.14(2.2 \%)$ with a standard deviation of 1426.23 .

\subsection{Optimal Recovery Time}

To the extent that the reactionary model is inconsistent with the strategic cycle, it is not sustainable in the long run; it is benefited by the lack of a constraint on the ending inventory each week tying it to the start-of-week inventory. The fact that there is a cost of recovery to strategic, but fortuitous deviations from long run optima is not sustainable gives rise to the question, "What is the optimal recovery path?" We conducted a sensitive analysis with respect to one dimension of this problem; given an initial surplus of inventory, how quickly should the inventory be reduced to the strategic optimum under different costs of inventory? We reformulated the singleweek model as a 6-week model with repeating demand pattern, thus we could remove the end of week constraint in the recovery model, allowing the number of days to return to strategic targets to be endogenous, rather than imposed by constraint by week's end.

Given an initial inventory of 100 units (in this example a shock which causes an excess inventory of 57 units over the strategic target of 43), we evaluated how many days pass before inventory returns to the optimal strategic targets. The answer depends on the cost of the inventory excess, and the opportunity cost of recovery. Figure 
4 shows the path to recovery under the various scenarios. In the low cost of inventory case (Invcost $\leq 5)$, the optimal recovery interval was 15 days; for higher inventory costs, the optimal recovery interval was 8 days. In every case, despite having the same total days to recovery, over the recovery interval, the deviation from the long run target inventory was smaller as inventory costs were higher.

\subsection{Multiple Supply Shocks}

To this point, we have considered a single deviation from strategic optimum, and the managerial options for adjusting to it. The far more common case is for repeated, daily deviations from planned inventory levels that result from regular deviations from planned supply of and demand for resources.

We designed and experiment with Poisson arrivals of demand around a daily mean demand. Similar to the optimal recovery path experiment, we generated instances of supply and demand over a six week period. We compared the repeating, strategic optimum inventories to the tactical level, reactive inventories in the face of random demand, as shown in Figure 5.

Because daily arrivals were random, optimal tactical deviations from planned inventory were pervasive and regular. More importantly, any cost incurred to regain the strategic target is likely in vain; subsequent supply and demand shocks essentially decimate any anticipated benefits of being on target. In this case, the strategic optimum targets provides even less value as a managerial target. Because a manager never knows what tomorrow will bring, efforts to manage to a target based on expectations prove costly and unrewarding. In short, in the face of regular and pervasive supply and demand shocks, strategic targets have little or no role in tactical decision making.

\section{Managerial Implications}

The managerial implications from this research are palpable. Strategic models like those described in the literature review such as $[8,13,14]$ propose strategic models as the basis for managing various fleets. However.front line decision makers who tend to be "short sighted"-maximizing current profits while eschewing future opportunities - may be more rational than the strategic modeling results would imply. Because of the real explicit and opportunity costs of managing to an inventory target, and the uncertainty of future conditions, a manager might rationally sacrifice uncertain future benefits for near term gain. In the case of multiple supply and demand shocks, not only is there a cost of recovery, but a successful recovery is not likely to improve future profit expectation.

Strategic vs tactical recovery inventory given starting inventory of 100

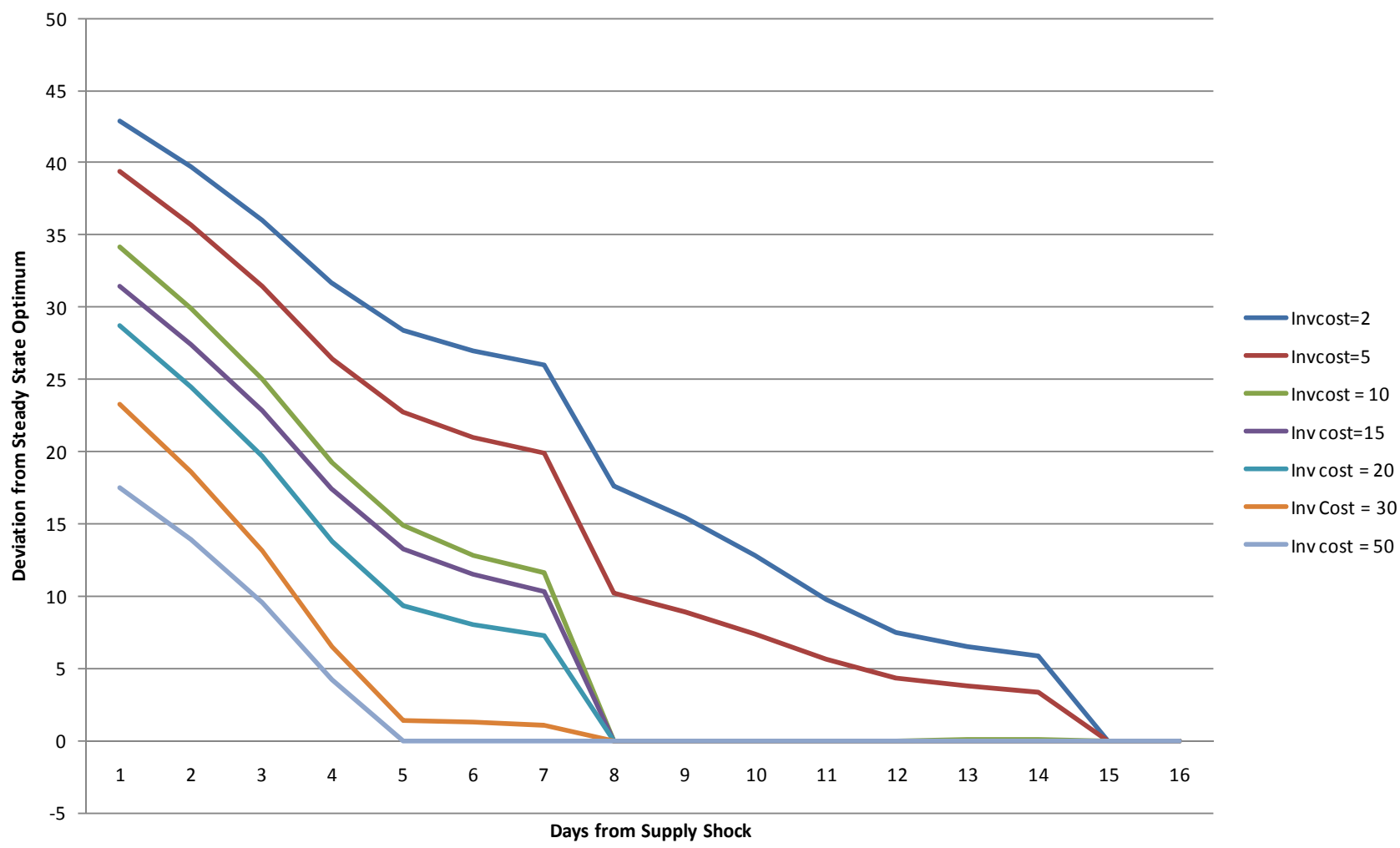

Figure 4. Strategic vs tactical recovery inventory given starting inventory of $\mathbf{1 0 0 .}$ 


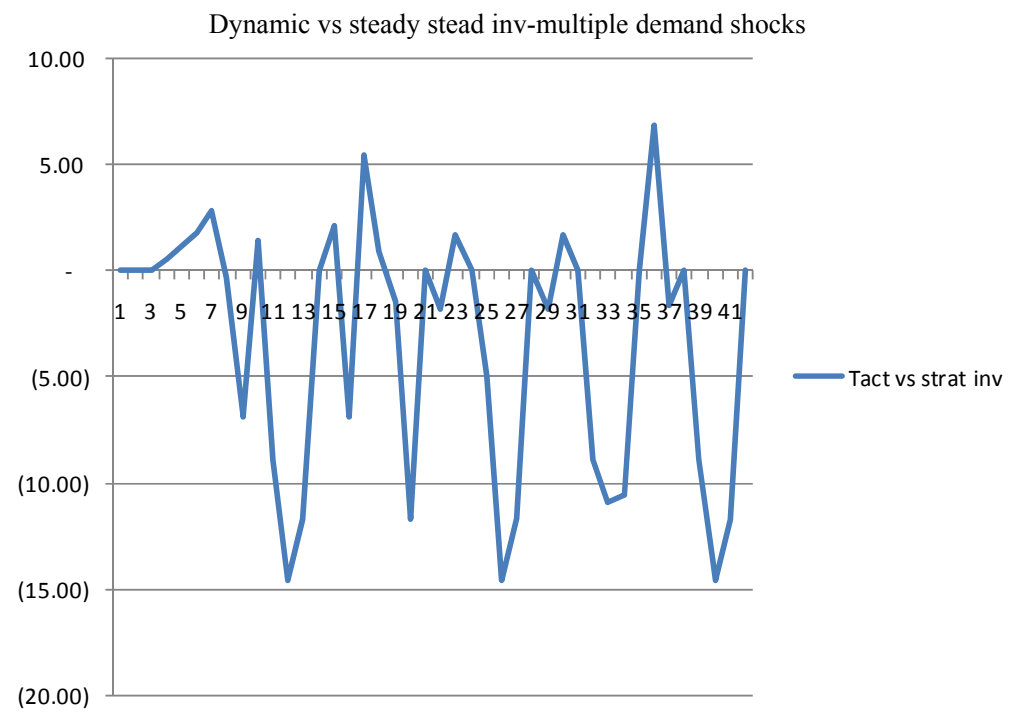

Figure 5. Dynamic vs steady stead inv-multiple demand shocks.

Simply, a manager should not be held accountable for managing fleet inventory relative to some long-term ideal or target.

\section{A Strategy for Combining Tactical and Strategic Models}

Given our finding that strategic model targets are not relevant in a tactical setting, and tactical model results may not be sustainable on a continued basis, what is the appropriate course of action? Simply, the strategic and tactical optimum inventories represent bounds on the optimal inventory. We suggest the optimal target inventory level rests in the interval between tactical and strategic optima; the actual optimum depends on the cost of inventory and the cost of adjustment. We suggest that rather than a target inventory level, a target inventory range is a better goal; the range is determined by the interval between the tactical optimum for each day (given current conditions), and the strategic optimum (given common long run conditions).

Alternatively, replicating the modeling horizon with tactical values in the first interval and strategic values in subsequent intervals allows the model to endogenously arrive at an optimal recovery period. Any use of either strategic or tactical modeling paradigm in isolation will likely lead to errant managerial action. It should be noted that any managerial action geared towards managing to that target is tempered by future uncertainty of supply and demand.

\section{Conclusions}

In this research, we evaluate the differences in strategic and tactical modeling paradigms with highly cyclical supply and demand patterns. While these modeling ap- proaches have both been widely used in the literature, the difference in the model recommendations and managerial implications have not been explored.

We find that in some cases, deviations from strategic optima may in fact be advantageous. That is, the strategic optimal target stock levels are not optimal in an execution setting. Thus, care should be taken when "managing to a target" that is well meant and derived optimally, but can be misdirected effort, actually creating additional costs and foregone profits.

Simply, we are faced with a paradox that strategic and tactical model recommendations do not necessarily match. While having and idea of strategically "where you want to be" is important to long run profitability and operational feasibility, managing too strictly to these targets can be shown to be suboptimal. Thus, a coordinated blend of the two approaches is required. We recommend an appropriate mix of the two model regimes: Each modeling paradigm sets a boundary on optimal operational parameters. Strategic models set long policy, tactical models to set optimal behavior given current conditions and the long run strategic targets. Any achieved value in this range is acceptable; which target to pursue more aggressively depends on the relative costs of adjustment and opportunity costs of straying from a strategic target.

This research focused on a single perturbation that drives a deviation from strategic targets; future research might examine more fully the managerial implications of persistent supply and demand shocks on the role strategic modeling in a highly stochastic setting.

\section{REFERENCES}

[1] J. Roy and T. Crainic, "Improving Intercity Freight Routing with a Tactical Planning Model," Interfaces, Vol. 22, 
No. 3, 1992, pp. 31-44. doi:10.1287/inte.22.3.31

[2] J. Cordeau, P. Toth and D. Vigo, "A Survey of Optimization Models for Train Routing and Scheduling," Transportation Science, Vol. 32, No. 4, 1998, pp. 380-404. doi:10.1287/trsc.32.4.380

[3] D. Huisman, L. Kroon, R. Lentink and M. Vromans, "Operations Research in Passenger Railway Transportation," Statistica Neerlandica, Vol. 59, No. 4, 2005, pp. 467-497. doi:10.1111/j.1467-9574.2005.00303.x

[4] M. F. Gorman, D. Sellers and D. Acharya, "CSX Railway Cashes in on Optimization of Empty Equipment Distribution," Interfaces, Vol. 40, No. 1, 2010, pp. 5-16. doi:10.1287/inte. 1090.0465

[5] M. F. Gorman, K. Crook and D. Sellers, "North American Freight Rail Industry Real-Time Optimized Equipment Distribution Systems: State of the Practice," Transportation Research Part C, Vol. 19, 2011, pp. 103-114. doi:10.1016/j.trc.2010.03.012

[6] W. B. Powell and T. A. Carvalho, "Real-Time Optimization of Containers and Flatcars for Intermodal Operations," Transportation Science, Vol. 32, 1998, pp. 110126.

[7] H. Sherali, E. Bish and Z. Xiaomei, "Polyhedral Analysis and Algorithms for a Demand-Driven Refleeting Model for Aircraft Assignment," Transportation Science, Vol. 39, No. 3, 2005, pp. 349-366. doi: $10.1287 /$ trsc. 1040.0090

[8] R. K. Ahuja, J. Liu, J. B. Orlin, D. Sharma and L. A. Shughart, "Solving Real-Life Locomotive-Scheduling Problems," Transportation Science, Vol. 39, No. 4, 2005, pp. 503-517. doi:10.1287/trsc. 1050.0115

[9] M. Lübbecke and U. Zimmermann, "Engine Routing and Scheduling at Industrial In-Plant Railroads," Transportation Science, Vol. 37, No. 2, 2003, pp. 183-197. doi:10.1287/trsc.37.2.183.15251

[10] Y. Ileri, M. Bazaraa, T. Gifford, G. Nemhauser, J. Sokol, and E. Wikum, “An Optimization Approach for Planning Daily Drayage Operations," Central European Journal of Operations Research, Vol. 14, No. 2, 2006, pp. 141-156. doi:10.1007/s10100-006-0165-6

[11] A. Erera, B. Karacık and M. Savelsbergh, "A Dynamic Driver Management Scheme for Less-than-Truckload Carriers," Computers\& Operations Research, Vol. 35, No. 11, 2008, pp. 3397-3411. doi:10.1016/j.cor.2007.01.019

[12] B. Vaidyanathan, K. Jha and R. Ahuja, "Multicommodity Network Flow Approach to the Railroad Crew-Scheduling Problem," IBM Journal of Research \& Development, Vol. 51, No. 3-4, 2007, pp. 325-344. doi: $10.1147 / \mathrm{rd} .513 .0325$

[13] M. F. Gorman, "Intermodal Pricing Model Creates a Network Pricing Perspective at BNSF," Interfaces, Vol. 31, No. 4, 2001, pp. 37-49.

[14] D. Adelman, "Price-Directed Control of a Closed Logistics Queueing Network," Operations Research, Vol. 55, No. 6, 2007, pp. 1022-1038. doi:10.1287/opre.1070.0408

[15] M. F. Gorman, "Hub Group Implements a Suite of OR Tools to Improve Operations," Interfaces, Vol. 40, No. 5, 2010, pp. 368-384. doi:10.1287/inte. 1100.0507

[16] A. Schaefer, E. Johnson, A. Kleywegt and G. Nemhauser, "Airline Crew Scheduling Under Uncertainty," Transportation Science, Vol. 39, No. 3, 2005, pp. 340-348. doi: $10.1287 / \operatorname{trsc} .1040 .0091$

[17] A. Jarrah, J. Goodstein and R. Narasimhan, "An Efficient Airline Re-Fleeting Model for the Incremental Modification of Planned Fleet Assignments," Transportation Science, Vol. 34, No. 4, 2000, pp. 349-363. doi: $10.1287 /$ trsc. 34.4 .349 .12324

[18] R. E. Hughes and W. B. Powell, "Mitigating End Effects in the Dynamic Vehicle Allocation Model," Management Science, Vol. 34, No. 7, 1988, pp. 859-879. doi:10.1287/mnsc.34.7.859 